\title{
Identidad cultural en grupos sociales de la zona fronteriza de Baja California
}

\author{
Ma. Luisa Rodríguez Sala \\ Instituto de Investigaciones Sociales \\ Universidad Nacional Autónoma de México
}

\section{Resumen}

En este trabajo se presenta un resumen comparativo de los resultados que se obtuvieron en el grupo de estudiantes y en el grupo de científicos y profesionistas en torno a la identidad cultural en la zona fronteriza. Tanto las conclusiones particulares como las generales comprueban las hipótesis que se sustentan en el marco teórico general de esta investigación, las que además se refuerzan con los subsecuentes resultados provenientes de un profundo análisis de los grupos sociales y del subgrupo de los adolescentes de conducta antisocial que complementan la investigación. El objetivo fundamental de esta primera etapa es lograr el primer acercamiento de carácter semiológico al entendimiento de la identidad cultural en una zona fronteriza del norte de México.

Palabras clave: identidad cultural, grupos sociales fronterizos, núcleos de identidad, elementos de identidad, adolescentes, características socio-lingüísticas.

\begin{abstract}
This document presents a comparative summary on the results obtained by a group of students jointly working with a group of scientists and professional people regarding the cultural identity in the border zone. The particular conclusions, as well as the general ones prove the sustained hypothesis in the general theoretical frame of this research, which also reinforce the resulting output. This output is the product of a profound analysis on the social groups of teenagers with anti-social behavior complementing the research. This first stage pretends to get the first outreach on the semiologic character to understand the cultural identity at a border zone from the North of Mexico.
\end{abstract}

Keywords: cultural identity, border social groups, identity nucleus, identity elements, teenagers, socio-linguistic characteristics. 


\title{
IDENTIDAD CULTURAL EN GRUPOS SOCIALES DE LA ZONA FRONTERIZA DE BAJA CALIFORNIA
}

\author{
Por \\ Ma. Luisa Rodríguez. Sala*
}

El estudio de la identidad cultural en la zona fronteriza mexicana ha constituido un tema de investigación sociológica de creciente interés por su relación con la actual situación de crisis que se presenta en México y que hace destacar precisamente la importancia de los elementos identificatorios a nivel nacional y regional. No es pues casual el que instituciones de investigación como el CFFNOMEX y el Instituto de Investigaciones Sociales de la UNAM, hayan realizado trabajos sobre esta temática, que si bien con enfoques teóricos y metodológicos diferentes, y a pesar de ello, coinciden en sus resultados generales y se complementan y refuerzan mutuamente.

En el Instituto de Investigaciones Sociales iniciamos la investigación desde 1981 y durante estos anos hemos ya concluido las etapas previstas y estamos presentando aqui parte de los resultados.

\section{MARCO TEORICO: EL ENFOQUE SEMIOLOGICO.}

La identidad cultural, de acuerdo al enfoque teórico de la investigación que reportamos, puede ser aprehendida en forma unitaria si se la considera integrada por un conjunto de sectores culturales todos ellos relacionados por un vínculo común que presenta tres vértices: interacción social, comunicación y relación experiencia-expresión que se expresan al través del uso de signos verbales y no-verbales, de aquí la importancia del aspecto scmiológico para el entendimiento de lo cultural. Es esta importancia la que nos ha llevado a postular que será el análisis del signo en su vertiente social lo que permitirá precisar los rasgos culturales que caracterizan la identidad de una comunidad, en nuestro caso, la fronteriza bajacaliforniana. Para lograrlo partimos, en nuestra investigación, del enfoque semiológico profundo e integral que hemos elaborado en el Seminario de Semiología del Instituto de Investigaciones Sociales de la UNAM. Aquí sólo haremos tres breves menciones de ese enfoque: 1) Partimos de un postulado básico: los símbolos sociales proceden de la realidad, son su reflejo e inciden en ella; 2) Nuestra concepción del signo destaca, para el estudio sociológico de la realidad, la vertiente programática que se identifica con una parte de la sociología, si se

\footnotetext{
*Ma. Luisa Rodríguez Sala, Mexicana. Maestra en sociología por la UNAM. Investigadora del lnstituto de Investigaciones Sociales de la UNAM. Entre sus obras puede mencionarse: El cientifico como productor y comu. nicador. El caso de Máxico. Parte del estudio que aquí se publica lo elaboró como investigadora visitante del CEFNOMEX en Tijuana, Baja California.
} 
la concibe como la dimensión que se ocupa de la relación entre los signos y sus usuarios $\mathrm{o}$, visto desde el otro lado, entre los societarios y los significantes que emplean y quc hacen posible la interacción y la comunicación sociales. 3) En el transfondo del problema del signo existe siempre la relación expcriencia-expresión. La experiencia lleva implícita, además, un conocimiento y una información que, a su vez, dan lugar a diferentes formas de expresión, que, como ya vimos, se modifican por la influencia de ciertas dimensioncs sociales que reflejan lo que sucede en la moderna sociedad científico-tccnológica. (Jiménez-Ottalengo, Rodríguez Sala y Paulín, 1980). En clla, cl individuo, en especial aquel que se encuentra en etapa vital de búsqueda de autoconciencia, sea por su edad (adolescentes), sea por su adaptación a un nuevo ambiente socio-cultural (migrantes), fincará su identidad con base en su propia experiencia, apartándose, en la gran mayoría de los casos, de las restantes formas de cxperiencias, y, con ello, está expuesto a una pérdida de su identidad cultural.

El incremento excesivo en las formas de la experiencia subjetiva, el apartarse de las experiencias colectivas y la existencia de sistemas persona lizados de valores sin relación con el sistema valorativo grupal (J. J. Smolicz, 1981), son elementos que intervienen y configuran los diferentes niveles del proceso de identidad de los individuos y de los grupos sociales. En su estudio se intentará un acercamiento que pueda llevar a la aprehensión de la identidad sociocultural, considerando que los valores medulares del grupo son experimentados, compartidos y expresados por sus miembros, quienes los seleccionan del conjunto almacenado en el sistema grupal, los organizan en un sistema personal que se adapta a sus propósitos e intereses y los expresan mediante cl cmpleo del sistema semiológico. De aquí que en el estudio empirico de la identidad sociocultural en algunos grupos sociales de la zona fronteriza bajacaliforniana, tratemos de determinar: a) las actitudes personales frente a los valores medulares del grupo social al que se pertencce y frente a los valores medulares de la sociedad global, y, b) las expresiones de comportamiento específicas ante $\mathrm{cl}$ uso o $\mathrm{cl}$ manejo de esos valores medulares, que serán los identificadores del grupo y de su identidad como comunidades distintivas, con sus rasgos culturales propios, pero tambićn con rasgos culturales supragrupales y que, finalmente, son los que permiten la integración de los grupos y las comunidades en una nación.

\section{ASPECTO EMPIRICO}

\section{Generalidades}

La investigación, en su primera etapa, incluye los grupos sociales de: a) Adolescentes, dividido en dos subgrupos: a) el de quienes han manifestado conductas antisociales y que representan las respuestas extremas a situacio- 
nes sociales de inadecuación en el tratamiento de problemas de innovación ${ }^{1}$, $y, b)$ el de quienes, con una conducta "normal" (no antisocial), representan un núcleo contrastante con el anterior: los adolescentes estudiantes. b) El segundo grupo lo hemos denominado operativamente, de Científicos y profesionistas y se constituye por quienes producen la ciencia-los científicosy por quienes se dedican a aplicar sus conocimientos al servicio directo de la sociedad - los profesionistas-. Ambos subgrupos forman parte del grupo ocupacional con el más elevado nivel de educación formal, y, por ello, con mayores posibilidades de asimilación de los procesos innovativos procedentes de la ciencia y tecnología y con un mejor manejo de la relación entre sistemas valorativos personales y sistemas valorativos grupales, $y$, supuesta y consecuentemente, con un nivel de identidad cultural elevado.

En este trabajo presentamos un resumen comparativo de los resultados que se obtuvieron en el grupo de estudiantes y en el grupo de científicos y profesionistas. Se analiza a los estudiantes que cursan cl tercer grado de instrucción media inferior (secundaria) en los diferentes tipos de escuelas del sistema educativo nacional y en los tres turnos en que funcionan estas escuelas. El universo cubierto en la ciudad de Tijuana cs de 550 alumnos, cifra que se integra con el $10 \%$ o proporcional a la población de cada uno de los ripos de escuelas; la edad de los alumnos ha quedado comprendida entre los 13 y 18 años.

En el grupo de científicos y profesionistas se ha cubierto, un universo que representa el 90\% (109 casos) del personal de investigación quien, durante principios de 1983 realizaba investigación científica, y, una muestra de 162 casos que representa del 10 al 15\% de los "Profesionistas" que ejercen su actividad en la ciudad de Tijuana.

Para cada uno de los dos grupos sociales se emplearon cuestionarios diferentes, que pretenden determinar los niveles de identidad a partir del empleo y uso de símbolos y de valores medulares. En este trabajo analizamos con mayor profundidad los resultados para el grupo de los estudiantes y buscamos su comprobación y ampliación en el grupo de científicos y profesionistas, que en cierta forma puede considerarse, en un corte longitudinal, como el futuro de parte de esos jóvenes por ahora estudiantes.

Las variables que se han seleccionado para ese trabajo, si bien constituyen sólo una parte del total de las que contiene el cuestionario utilizado, son representativas del núcleo de identidad, que se ha precisado al través de una serie de preguntas que se agrupan, para los estudiantes, en tres nú-

1 Este subgrupo (menores de conducta antisocial) es investigado por la investigadora Leticia Ruiz de Chávez del L.I.S. UNAM. 
cleos o conceptos: a) Concepto de "IIćroes y personajes" (H. y P.), b) Concepto de "Patria" (P.), y, c) Concepto del "Nosotros" y "Los otros".

Para los Científicos y Profesionistas los conceptos que han conformado la identidad se integran en tres núcleos: valores medulares, clementos de identidad y símbolos sociales.

Cada uno de estos núcleos constituye el punto de partida para señalar y determinar la influencia que ejercen los factores considerados determinantes de la identidad y que se expresan por las siguientes variables independientes: 1) Factor secuencial de asentamiento en la zona, se obtuvo al través de los indicadores: lugar de nacimiento y número de años de residencia en la zona del encuestado y de sus padres. El factor indica la existencia de tres secuencias: a) encuestado y padres nacidos y radicados en la zona frontcri$z a, b)$ encuestado y padres nacidos en la zona fronteriza o fuera de clla, pero con más de 10 años de residencia aquí, y, c) encuestado y padres no nacidos en la zona y con mènos de 10 años de residir en ella. 2) La segunda variable responde a la relación fronteriza a través de algunos aspectos laborales de la familia del encucstado. 3) La tercera variable precisa la influencia de los medios masivos de comunicación, los indicadores considerados son: canales y programas de televisión y periódicos y sus secciones. Las preferencias por estos medios masivos establecen cuatro grupos de respuestas que señalan una influencia creciente de los medios de comunicación norteamericanos frente a los nacionales. 4) La cuarta variable, o en este caso factor, está dado por el tipo de escuela en que estudian los adolescentes y que son: particulares, por cooperación, oficiales diurnas y oficiales vespertinas y nocturnas.

Los últimos tres factorcs son exclusivos del grupo de Estudiantes, en tanto que el primero corresponde a los dos grupos sociales hasta ahora investigados en esta zona.

\section{Conformación de los núcleos de identidad.}

En el grupo de estudiantes el concepto de héroes y personajes se formó con dos conjuntos de preguntas, en el primero se manejaron a los héroes y personajes se formó con dos conjuntos de preguntas, en el primero se manejaron a los héroes como símbolos de ideales individuales o de aspiraciones colectivas, y, el segundo se integró con los personajes y las celebridades, quienes temporalmente pueden también llegar a convertirse en símbolos, cspecialmente de los valores grupales. Con ambos símbolos el adolescente, precisamente por el apremio propio de su etapa evolutiva, busca la autoidentificación al adoptar un modelo imitable que selecciona entre la gama de símbolos que la socicdad le provee. Este anhelo de identificación opera social- 
mente como fuerza culturalmente unificadora, de aquí que la selección de héroes y celebridades y la identificación con alguno de cllos ha constituido parte de este trabajo. Para lograrlo se pidió al estudiante mencionar, primero, cuando menos, tres nombres de los héroes que más admira, y posteriormente, señalar como quién de ellos les gustaría ser; y, en otra pregunta, indicar su personaje (celebridad) preferido en cuatro áreas de cultura general (historia, política, literatura y ciencia) y en cuatro actividades de diversión (música, cine, televisión y deportes). Las respuestas a estos dos conjuntos de preguntas dieron cuatro niveles de identidad, el más alto de ellos ha correspondido a las respuestas en que se seleccionaron héroes y personajes exclusiva o preponderantemente nacionales, los siguientes niveles incorporan sucesivamente mayor número de héroes y personajes extranjeros o universales y disminuyen la proporción de mexicanos hasta llegar al cuarto y último nivel en el que el mayor número de héroes y personajes exclusiva o preponderantemente nacionales, los siguientes niveles incorporan sucesivamente mayor número de héroes y personajes extranjeros o universales y disminuyen la proporción de mexicanos hasta llegar al cuarto y último nivel en el que el mayor número de héroes y personajes seleccionados son extranjeros o universales.

El segundo núcleo de identidad - concepto de "Patria"- lo determinamos al través de aquellas preguntas que involucran la relación directa con México, mediante reacciones, preferencias y representaciones del país mismo. Las preguntas consideradas han sido las siguientes. "Cuando te mencionan la palabra México, ¿Qué es lo primero que se te viene a la mente?; "Anota los tres países que más admiras"; "¿Qué es lo que más te gusta de tu patria:", "¿Qué es lo que más te disgusta de tu patria:", y, "Anota tres formas de representar a la patria".

Las respuestas a cada pregunta fucron agrupadas en cuatro niveles que van de mayor a menor arraigo nacional según la calificación obtenida con base en una puntuación decreciente.

FI tercer núcleo de identidad lo hemos seleccionado siguiendo a Gurvitch y corresponde al conocimicnto del "nosotros" y de los otros, que aplicado en su connotación extrema identificaría al "nosotros" con lo nacional y lo regional y al "los otros" con lo extranjcro. En una zona fronteriza las connotaciones de este tipo de conocimiento adquicren importancia especial en su relación con la identidad nacional, ya que precisamente en el contacto y enfrentamiento cotidiano de los dos términos se produce lo que hemos llamado la relación de doble vía que fortalece $y$ acrecienta lo nacional al confrontarlo con la extranjero, en un proceso de transculturación que se manifiesta al través de diversos niveles de identidad nacional. Estos niveles, en el mejor de los casos - niveles más elevados- preserveran lo "nuestro" con re- 
chazo de los "otro"; en el nivel intermedio, se conserva lo "nacional" y se incorpora lo que de "los otros" resulta conveniente desde la perspectiva transcultural; y, por último, en el nivel más bajo de identidad, se asimila lo extranjero en un proceso de "nepantlismo" que abre el camino a la transformación de los valores de lo "nucstro" y la formación de una cultura en peligro en la zona fronteriza bajacaliforniana. Las preguntas que hemos analizado para este concepto de identidad son las que se refieren a: "¿Quiénes son para tí los extranjeros?" y "¿Qué es lo que más te gusta y te disgusta de: los extanjeros, tus compatriotas y los bajacalifornianos?" (dos preguntas para cada categoría). Las respuestas, una vez que se confirmó un indudable conocimiento de lo que se entiende por el término "extranjero", fueron procesadas en tal forma que permiten establecer comparaciones entre las dos modalidades, integrándose tres niveles de identidad: a) Predominio del "Nosotros" sobre "Los Otros", b) Estabilidad o equilibrio entre el "Nosotros" y "Los Otros", y, c) Predominio de "Los Otros" sobre el "Nosotros".

Para el grupo de científicos y profesionistas la identidad se integró al través de tres conjuntos de preguntas: a) Valores medulares que se constituyen en mecanismos o formas de mantener la integridad cultural y con ello la identidad nacional. Se han cubierto tres ámbitos de la sociedad: el familiar, el profesional y el cívico. En esta presentación no se han considerado los resultados de este núcleo de identidad. El segundo conjunto es el que corresponde a los elementos de identidad que actúan como mecanismos específicos que favorecen esa identidad. Los quince elementos que hemos incluido en el instrumento proceden de los que se mencionaron en la prueba piloto como los más significativos para el mantenimiento de la identidad en la zona fronteriza.

Con el objeto de determinar cuáles de ellas son las que contribuyen para preservar la identidad, se pidió a los entrevistados seleccionar los cinco más importantes y contrastar con los tres sin influencia.

La elaboración de las respuestas ha implicado una serie de matices hasta llegar a obtener dos resultados complementarios:

En primer lugar se ha valorado cada elemento individualmente al considerar el total de frecuencias o selecciones con que se ha visto favorecido y que representa la importancia que el grupo le concede en el conjunto de la totalidad de elementos. Posteriormente se han precisado las combinaciones de los elementos seleccionados para lo que se hizo necesario agruparlos en categorías temáticas afines.

Los elementos de identidad que la preservan y que han sido seleccionados individualmente son tomados en cuenta siempre y cuando el porcen- 
taje de frecuencias recibidas sea superior o cercano al 500/o, puesto que se ha considerado cada elemento en relación a la preferencia también de cada entrevistado y la dispersión de frecuencias, debido al elevado número de elementos, resulta de importancia estadística. Precisamente esta dispersión de frecuencias señala que entre la gama de los elementos que preservan la identidad, se repiten constantemente algunos de ellos y se descartan los restantes, también es relevante el hecho de que los elementos que se repiten aparecen en todos los subgrupos con porcentajes similares.

El tercer núcleo de identidad es el de los símbolos que proporcionan la imagen que del sistema cultural se forman sus miembros y consideramos que podemos hablar de un elevado nivel de identidad cuando se comparte un sistema similar de símbolos. Es por esta razón que se elaboró una lista de símbolos procedentes de la exploración previa- y se pidió señalar los más importantes, primero como identificadores de todos los mexicanos, después los que han sido adoptados íntegramente por los originarios de la zona o con más de 2 años de residencia, y por último los modificados a la situación propia de quienes son de reciente asentamiento en la zona.

Dentro de este mismo núcleo se precisó un subnúcleo, el sociolingüístico, que se refiere al uso y manejo del idioma en su relación con lo social. Al determinar algunas características en relación con el uso del idioma inglés, confirmamos, por un lado, la importancia del elemento lingüístico en el fortalecimiento de identidad y, simultáneamente el manejo que se requiere del idioma de la sociedad colindante, tanto por el entrevistado mismo como por los miembros de su familia. En este núcleo se interrogó también acerca del uso del inglés en el desarrollo de las actividades profesionales y de su empleo en el ámbito de la vida familiar.

\section{RESULTADOS}

Los conceptos de identidad que hemos descrito fucron pucstos en relación con las variables independientes que corresponden a cada grupo social, los resultados de csas relaciones constituyen los diferentes apartados de la investigación completa. En csta ponencia se exponen los resultados más relevantes en forma sumaria y a manera de conclusiones provisionales que se encuentran ampliadas y justificadas en el informe final.

En primer término se establece en qué núcleos de identidad se conforma una relación y con cuál o cuáles de las variables; en el subgrupo de los estudiantes encontramos que:

1. Para los tres núcleos que integran el concepto de identidad cultural en la ciudad de Tijuana, de los cuatro factores determinantes, sólo dos de ellos 
son los que influyen decisivamente: el factor de asentamiento secuencia en la zona y cl tipo de escuela.

El factor constituido por los medios de comunicación ejerce su influencia para los núcleos de "Héroes y personajes" y de "Patria" y no lo hace en e. núcleo del "Nosotros y los otros",

El tercer factor, el de las relaciones fronterizas, no resultó significativo en ninguno de los tres núcleos de identidad, por lo que se descarta para este análisis.

Para los científicos y profesionistas el factor de asentamiento secuencial sí ha marcado diferencias en la expresión de la identidad y en el análisis detallado de este grupo, los científicos generalmente se distinguen en sus opiniones de las que emiten los profesionistas.

El cómo se manifiesta la influencia de los factores en cada núcleo de identidad se precisa en las siguientes conclusiones:

2. El factor de asentamiento secuencial en la zona señala que la identidad cultural que se simboliza al través de los "Héroes y personajes", decrece en su nivel a medida que se permanece mayor tiempo en la zona y esto es válido -estadísticamente-- para los alumnos de escuelas particulares y, en menor proporción, para los de las escuelas oficiales. De ello se puede inferir que, dentro de una identidad básicamente nacional, los matices más transculturados corresponden a los adolescentes que ocupan una mejor posición sociocconómica y tienen, además, un más elevado factor de ocupación en la zona.

Por lo que corresponde al núclco de "Patria", cste factor cs significativo para los alumnos de las escuclas oficiales y para los de las particulares; en los dos conjuntos se marca claramente la influencia del factor tiempo, ya que: a menor tiempo de residir en la zona, mayor nivel de identidad nacional.

En el tercer núcleo de identidad ("Nosotros" y "Los otros") el factor vuelve a establecer distinción entre las escuelas particulares y las oficiales. Los datos indican dos hechos significativos: a) Los originarios de la zona y quienes tienen un elevado factor de asentamiento en ella, son quienes muestran, por un lado, un nivel de identidad que se distingue por una estabilidad entre cl "Nosotros" y "Los otros", pero, al mismo tiempo con una aceptación de lo extranjero que predomina sobre lo nacional y que es más alto, numéricamente hablando (78\%) en las escuelas particulares que en las oficiales $(66 \%)$. b) Quienes han llegado a residir a la zona en ćpoca más re- 
ciente son quienes conservan una identidad con predominio del "Nosotros" sobre "Los Otros" en una proporción que es del $33 \%$ en las escuelas particulares y del $490 \%$ en las oficiales.

Como conclusión final derivada de la influencia del factor de asentamiento secuencial en la zona fronteriza podemos establecer que:

3. A mayor número de años de residencia en la zona, mayor predominio de los niveles de identidad que marcan, dentro de una identidad básicamente nacional, una tendencia hacia la transculturación y aceptación progresiva de celebridades, personajes y héroes extranjeros y de una identificación, también progresiva, con los "Otros, sea mediante una manifiesta admiración por "los extranjeros", sea por una estabilidad o equilibrio entre lo "nacional y regional" y lo "extranjero" propia de toda zona fronteriza. Con ello se comprueba que la transculturación obedece indudablemente a un proceso de doble vía en el cual se acepta lo de "Los Otros" en un auténtico sentido fronterizo que no rechaza irracionalmente lo ajeno, sino que, evaluándolo, lo incorpora e integra en una sensata apropiación que, simultáneamente conserva lo propio y lo expresa por medio de los símbolos sociales y patrios que constituyen un sistema simbólico individual, que, en este caso, concuerda con el grupal y que permite hablar de una identidad cultural nacional con los matices propios de transculturación normal a toda zona fronteriza.

4. El factor que corresponde a los medios de comunicación determina diferentes niveles de identidad en los núcleos de "Héroes y personajes" y en el de "Patria". En cuanto al primero es evidente la relación entre la identidad y los medios masivos de comunicación que prefieren los adolescentes. Nuevamente se observa una diferenciación por tipo de escuela, ya que los estudiantes de las particulares se ubican preferentemente en los niveles más bajos de identidad cultural, destacándose un incremento en el número de estudiantes cuando el factor representado por los medios de comunicación indica, a su vez, una elevada influencia de medios extranjeros combinada con una escasa lectura de periódicos locales y/o nacionales. No sucede esto con los alumnos de las escuelas oficiales, entre quienes la relación es evidente, ya que al preferir medios de comunicación preponderantemente nacionales seleccionan también "héroes y personajes" que señalan una elevada identidad nacional, y, cuando los medios de comunicación representan una mezcla de la programación nacional y la extranjera, reforzada por la lectura de periódicos nacionales, entonces, la proporción de los símbolos sociales se inclina por una selección de "héroes y personajes" universales o extranjeros y una disminución en la selección e identificación con los nacionales. 
Los medios de comunicación también contribuyen a fijar un nivel de identidad en el núcleo de "Patria" y en tres de los cuatro tipos de escuela; son los alumnos de las escuelas por cooperación los únicos entre quienes no se da relación entre los dos factores. La relación, ctitre los alumnos de las escuelas particulares y oficiales comprucba, en términos generales, que: a mayor predominio de los medios masivos preponderantemente nacionales, se alcanzan, al traves del concepto de "patria", también niveles de identidad nacional más elevados y que a medida que los medios masivos preferidos por los estudiantes se tornan extranjerizantes o transculturados, su identidad patria se desliza y las frecuencias más elevadas quedan localizadas en los niveles más bajos de identidad.

La conclusión final que corresponde al factor de los medios de comunicación masiva en la ciudad de Tijuana relacionado con el concepto de identidad cultural indica que.

5. Sin lugar a dudas los medios de comunicación masiva se constituyen, en esta zona fronteriza del país, en uno de los vehículos más poderosos que contribuyen a fomentar una progresiva pérdida de identidad, pérdida que podría contrarrestarse con una adecuada política cultural del estado, con especial énfasis en las ciudades fronterizas, $y$, en ellas con atención especifica a los medios de comunicación, incrementando la programación cultural nacional al través de la creación y fomento de canales televisivos locales, la distribución eficiente de periódicos y publicaciones de circulación nacional, y, en general, el mejoramiento de las actividades culturales locales.

Estc reforzamiento cultural, impostergable en la zona, seria congruente con la política cducativa que el Estado ha implementado durante ya un largo período al inculcar en los estudiantes los símbolos sociales, $y$, en especial, los patrios, que, de acuerdo a los resultados de esta investigación, son los que preservan la identidad cultural y se sustentan en el conocimiento histórico que se transmite vía el sistema educativo nacional.

Entre los científicos y los profesionistas los elementos de identidad que han destacado a través de nuestra investigación indican que:

6. En primer lugar, se establece una distinción de opiniones entre los dos subgrupos: científicos y profesionistas. Los miembros del primero han seleccionado los siguientes cuatro elementos: "Conocimiento de nuestro pasado histórico", "El incremento de la comunicación con el resto del país", "El empleo adecuado y cuidadoso, en el ámbito familiar, del idioma castellano", y, "La observancia de las tradiciones populares nacionales". Los han mencionado en un orden decreciente de importancia porcentual que va del $880 /$ o al $50 \%$ o. 
El subgrupo de profesionistas se divide a su vez, en dos subconjuntos: abogados, médicos e ingenieros por un lado, y por el otro, contadores públicos. En las tres primeras profesiones, sus miembros seleccionan, con el mayor número de frecuencias, al igual que los científicos, al elemento de identidad que habla de "El conocimiento de nuestro pasado histórico", seguido por "El incremento de la comunicación con el resto del país", por "El empleo del idioma castellano", $y, \mathrm{cl}$ cuarto elemento ha sido, para este subgrupo el que se refiere a "El control social sobre niños y jóvenes a fin de que observen las costumbres y tradiciones nacionales. Los contadores públicos han seleccionado los mismos clementos, pero en un diferente orden de prioridades ya que el más clevado número de preferencias ha sido para el elemento quc asigna al idioma castellano un uso correcto y el que obtuvo el menor número de selecciones es el relativo al conocimiento del pasado histórico.

7. Sin considerar el orden de preferencia, la totalidad de los miembros del grupo ha coincidido en la selección de tres elementos de identidad que representan, a su vez, tres núcleos diferentes: el histórico, el sociolingüístico y comunicativo, y que además, representan simultáneamente los lazos de unión nacional. El conocimiento de nuestro pasado histórico enlaza lo actual con el pasado y hace posible el entendimiento de nuestra identidad nacional con base en lo que sucedió a nuestros antepasados y en lo que ellos nos han legado. Fste conocimiento adquiere especial relevancia en una zona fronteriza en donde el particular pasado histórico señala las diferencias fundamentales frente a los vecinos fronterizos con un pasado histórico nacional diferente.

El empleo del idioma castellano adecuada y cuidadosamente en el ámbito familiar y social, constituye la marca distintiva, el símbolo social por excelencia que diferencia a los habitantes de dos paises con un transfondo lingü ístico diferente.

El tercer elemento, el de matiz comunicativo externo (medios de transporte, programas televisivos y radiofónicos) representa una necesidad particular de los residentes de la zona geográfica más alcjada del centro de decisiones en un sistema político centralista por excelencia.

El cuarto elemento de identidad, si bien diferente en su expresión para cada subgrupo, pertenece, en los dos casos, al mismo núcleo: el sociocultural, que representa aquellos elementos relacionados con las costumbres y tradiciones, así como con las actividades artísticas y deportivas, que, a su vez, son producto del ambiente sociocultural de una sociedad dada. Se debe hacer resaltar el hecho de que en tanto los científicos seleccionaron el elemento que se refiere a "Observancia de tradiciones populares", los profesionistas dieron el $54 \%$ o de preferencias al elemento que lleva en si un in- 
grediente de control social, precisamente sobre los miembros jóvenes de $l_{2}$ familia, con lo que se marca nucvamente la importancia que el ámbito familiar representa en la conservación de la identidad nacional.

En el análisis de los cinco elementos de identidad que cada individuo seleccionó como los más importantes, se da una combinación de elementos pertenecientes a diferentes aspectos, repitiéndose constantemente lo que hemos mencionado en párrafos anteriores, $y$, consecuentemente las combinaciones que reciben el más elevado porcentaje, son aquellas en que aparecen mezclados los elementos histórico, sociocultural y de comunicación, con lo cual se confirma la conclusión fundamental de este inciso, y que, brevemente expuesta, indica que:

Los elementos más importantes para preservar la identidad en la zona fronteriza se ubican en lo histórico, en lo social y en la comunicativo, con fuerte apoyo al clemento sociolingüístico que se manifiesta por el uso correcto del idioma castellano.

8. Los símbolos que han obtenido - en una ordenación de mayor a menor importancia- los primeros tres lugares como identificadores de todos los mexicanos, se localizan, para el subgrupo de científicos, en cuatro de los siete símbolos que se enlistaron para ser seleccionados, y son: idioma, símbolos patrios, costumbres y tradiciones e idiosincracia; se seleccionaron del 50 al 7o lugar: los signos somáticos y el pasado histórico; el símbolo religioso (culto y festividades religiosas) obtuvo el 4o lugar que representa, en esta lista de ordenación el sitio de indiferencia como identificador de todos los mexicanos.

En el subgrupo de profesionistas sus miembros otorgan preferencias en los tres primeros lugares a las mismas expresiones simbólicas, la diferencia con los científicos radica exclusivamente en la proporción que corresponde a cada símbolo. En este subgrupo es el símbolo que representa el aspecto histórico el que se considera indiferente y los lugares más bajos ( 50 al 70 ) quedan ubicados en los símbolos de religión y en los signos somáticos.

Al considerar la adopción y modificación de símbolos, destaca la conclusión siguiente:

9. Se adoptan aquellos símbolos y características que también son los que identifican a los mexicanos y, que, por lo tanto, tienen una validez nacional como son los símbolos patrios, el idioma y las costumbres y tradiciones.

Cuando se trata de modificación de símbolos quedan sujetos a ella, fundamentalmente los que, por su carácter social son susceptibles de transforma- 
ción por quienes, con un bajo factor de asentamiento secuencial en la zona, se ven sujetos a la influencia del nuevo ambiente sociocultural que repercute o facilita el modificador las costumbres y tradiciones, el idioma y las características de comportamiento, actitudes y formas de pensar que es lo que hemos designado como idiosincracia.

Los símbolos patrios representan los caracteres de identidad con el menor grado de transformación y se constituyen así en los símbolos más cstables de la identidad cultural que encontramos, no sólo expresados en la selección y autoidentificación con héroes de la historia, entre los adolescentes estudiantes, y en su referencia a los colores patrios, a la bandera, al himno, al escudo como símbolo identificatorios de la "Patria", sino que se manifiestan gráficamente en los murales que otro grupo de adolescentes, los cholos de Tijuana, utilizan como la expresión de su identidad grupal frente a la sociedad global. ${ }^{2}$

El último aspecto que se relaciona directamente con la identidad lo constituye el enfoque sociolingúistico que caracteriza al grupo social que radica en una zona de natural intercambio lingúístico y que permite determinar algunas características en relación al uso del idioma inglés que confirman, la importancia del elemento lingü ístico en el fortalecimiento de la identidad, y simultáneamente el manejo que se requiere del idioma de la socicdad colindante por el entrevistado y por los miembros de su familia, así como el uso del inglés en el ámbito profesional y en el ámbito familiar. Fsta informa ción fué investigada solamente en el grupo de los "Científicos y Profesio nistas" y confirma, por un lado, resultados de otras investigaciones nues tras relativas a los "Científicos" y por otro, lo que en el CEFNOMEX se ha investigado en relación al uso del idioma inglés en la zona fronteriza del norte de México. men en:

Las principales conclusiones de este apartado sociolingüístico se resu-

10. Los investigadores, como ya se ha establecido en otros trabajos sobre sociología de la actividad científica en México, están relacionado con el sistema científico internacional tanto por los estudios realizados en el extranjero como por su manejo del inglés, el idioma científico de nuestra época y por otras actividades académicas (asistencia a eventos y publicación de trabajos). El contraste entre entre los científicos y los profesionistas es evidente y se expresa por un mejor manejo del inglés entre los miembros que se ubican en el área disciplinario de las ciencias exactas y naturales frente a quienes trabajan en el campo de las ciencias de lo humano. lise mejor mane-

\footnotetext{
2Véase: "El cholismo en Tijuana", Manuel Valenzucla, José Cuéllar y Laura Cummings. (Investigación en pro ceso). (EFNOMEX.
} 
jo del inglés se manifiesta al través del empleo de términos en ese idioma durante el desarrollo del trabajo profesional y es así como el subgrupo que requiere un empleo intensivo del inglés es el de los investigadores en ciencias exactas $(74 \% \%)$, quienes continua o diariamente los emplean en las otras dos profesiones de esta misma área científica: profesionistas en ciencias de la salud y de la construcción sus miembros también hacen uso continuo de terminología inglesa, pero en porcentajes menos elevados, $45 \%$ y $52 \%$ respectivamente. Los científicos que se desempeñan en ciencias de lo humano, son quienes, en este subgrupo de disciplinas, dan el mayor porcentaje de dominio completo del inglés lo que está en relación con un requerimiento en su trabajo profesional continuo o diario de los términos en el idioma de referencia. Las otras dos ocupaciones humanas - en el sentido disciplinarioabogados y contadores públicos-emplean ocasionalmente los términos en inglés y de ahí que su manejo sea también superficial e incipiente. Las profesiones que ellos ejercen están dirigidas a cubrir servicios a empresas o individuos que se ubican en una jurisdicción local, regional o nacional, en tanto que quienes ejercen en ciencias de la salud y la construcción en esta zona fronteriza, atienden con frecuencia a personas procedentes del pais vecino, algunos de ellos con escaso mancjo del español. Estas situaciones son especialmente frecuentes para los dentistas y médicos, quienes, por un lado cuentan con una clientela fija que procede de la zona fronteriza norteamericana y del interior de ese país, y por otro lado, por la indole de sus ocupaciones, frecuentemente asisten a cursos y reuniones académicos en centros hospitalarios y científicos de los Fstados Unidos de América.

11. En el ámbito familiar el uso de términos en inglés representa una característica sociolingüística propia de una zona fronteriza, su cmpleo es incvitable en el intercambio constante de información y bajo la influencia de los medios masivos de comunicación; de aquí que, en el conjunto grupal estudiado, quienes tienen el mayor nivel de manejo del idioma inglés y lo emplena con más frecuencia en el desarrollo de su profesión - los científicosson también quienes dan los mayores porcentajes $(29 \%$ ) de una ausencia total de uso del idioma extranjero en el ámbito de las relaciones familiares. Es necesario señalar que se exceptua de lo anterior a los profesionistas en ciencias de la salud, quienes han señalado que, en la gran mayoría $(91 \%$ o) los miembros de su familia emplean diario y ocasionalmente términos en idio$\mathrm{ma}$ inglés. En las restantes profesiones -abogados, ingenieros y contadores públicos - el uso diario y ocasional representa un $82 \%$ y el no empleo de los términos en idioma inglés, representa el $18 \%$ o del total de casos.

A pesar de este empleo alto del inglés en el ámbito profesional y en el familiar, la valoración del uso del idioma español como un elemento y como un símbolo de mantenimiento de la identidad cultural ha ocupado lugares importantes para los dos grupos sociales que hemos estudiado. De aquí que, también con apoyo en los resultados de la investigación realizada por el CEFNOMEX sobre aspectos del uso del español, se concluye que: 
12. El aspecto sociolingü ístico -manejo y uso del idioma en relación con lo social que senala una fuerte influencia del idioma inglés, se explique en función del tipo de actividad que se desempeña mas que de la expresión de una paulatina pérdida de identidad por influencia del idioma extranjero y que este uso del mismo, se compensa con la expresión simbólica que ha asignado, en los dos grupos, al idioma español un elevado rango en los niveles de identidad cultural.

A manera de resumen final y de acuerdo a los resultados comentados y a las conclusiones derivadas de ellos, se puede comprobar la existencia de niveles diferenciales de identidad de acuerdo, primeramente al factor de asentamiento secuencial en la zona y después a los diferentes contextos socioeconómicos de procedencia, en especial entre el subgrupo de los estudiantes y que verifica y refuerza parte de los resultados de la investigación del CEFNOMEX. Por otra parte, al localizar ciertas diferencias en las opiniones de los científicos frente a las de los profesionistas, y, aún entre las diferentes profesiones, se destaca otro factor más: a la actividad ocupacional que marca diferentes niveles de identidad para quienes ejercen diferentes actividades.

Tanto las conclusiones particulares como las generales, comprueban las hipótesis que se sustentan en el marco teórico general de esta investigación, las que además se refuerzan con los subsecuentes resultados que provienen de la profundización en el análisis de los dos grupos sociales así como de aquellos resultados que procederán del subgrupo de los adolescentes de conducta antisocial y que complementa la investigación en esta su primera etapa que ha tenido como objetivo fundamental el lograr el primer acercamiento de carácter semiológico al entendimiento de la identidad cultural en una zona fronteriza del norte de México.

\section{BIBUOGRAFIA}

BELL, Daniel. "The disjunction of culture and social structure: some notes on the meaning of social reality" en: science and Culture: A study of cohesive and disjunctive forces. Edited by Gerald Holton, Houghton Mifflin Company, Boston, The Riverside Press, Cambridge, 1965'

DUBOS, Rene. Reason awake: Science for man, Colombia University and London, 1970.

IIMENEZ-Ottalengo, R. Rodríguez Sala, M.L. y Paulin. G. "Reflexiones acerca de la semiología" en: Lecturas de Semiologia, Facultad de Ciencias Políticas y Sociales e Instituto de Investigaciones Sociales, UNAM. México, 1980.

SKOLIMOWSK1, Henry. "The scientific world view and the illusions of progress" en: Social Research, Vol. 41. Núm. 1, Spring, 1974.

SMOLICZ, J.J. "Core values and cultural identity" en Ethnic and Racial Studies, Vol. 4, Number 1, January 1981. 